\title{
Sex differences in outcomes of heart failure in an ambulatory, population-based cohort from 2009 to 2013
}

\author{
Louise Y. Sun MD SM, Jack V. Tu MD PhD, ${ }^{\star}$ Thais Coutinho MD, Michele Turek MD, Fraser D. Rubens MD MSc, \\ Lisa McDonnell MSc MBA, Heather Tulloch PhD, Anan Bader Eddeen MSc, Lisa M. Mielniczuk MD MSc
}

Cite as: CMAJ 2018 July 16;190:E848-54. doi: 10.1503/cmaj.180177

Visual abstract available at www.cmaj.ca/lookup/suppl/doi:10.1503/cmaj.180177/-/DC2

\begin{abstract}
BACKGROUND: Heart failure remains a substantial cause of morbidity and mortality in women. We examined the sex differences in heart failure incidence, mortality and hospital admission in a population-based cohort.
\end{abstract}

METHODS: All Ontario residents who were diagnosed with heart failure in an ambulatory setting between Apr. 1, 2009, and Mar. 31, 2014, were included in this study. Incident cases of heart failure were captured through physician billing using a validated algorithm. Outcomes were mortality and hospital admission for heart failure within 1 year of the diagnosis. Probability of death and hospital admission were calculated using the
Kaplan-Meier method. The hazard of death was assessed using a multivariable Cox proportional hazard model.

RESULTS: A total of 90707 diagnoses of heart failure were made in an ambulatory setting during the study period (47\% women). Women were more likely to be older and more frail, and had different comorbidities than men. The incidence of heart failure decreased during the study period in both sexes. The mortality rate decreased in both sexes, but remained higher in women than men. The female age-standardized mortality rate was 89 (95\% confidence interval [Cl] 80-100) per 1000 in 2009 and 85 $(95 \% \mathrm{Cl} 75-95)$ in 2013 , versus male age- standardized mortality rates of $88(95 \%$ $\mathrm{Cl} 80-97)$ in 2009 and 83 (95\% Cl 75-91) in 2013. Conversely, the rates of incident heart failure hospital admissions after heart failure diagnosis decreased in men and increased in women.

INTERPRETATION: Despite decreases in overall heart failure incidence and mortality in ambulatory patients, mortality rates remain higher in women than in men, and rates of hospital admission for heart failure increased in women and declined in men. Further studies should focus on sex differences in healthseeking behaviour, medical therapy and response to therapy to provide guidance for personalized care.

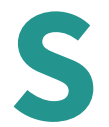

ubstantial progress has been made in the management of heart failure over the past 10 years. ${ }^{1}$ However, heart failure remains a common cause of morbidity and mortality, with an estimated prevalence of $1 \%-2 \%$ in the general population and a lifetime risk of 1 in 5 in men and women., ${ }^{2,3}$ Heart failure accounts for $35 \%$ of total cardiovascular mortality in women. ${ }^{4}$ Compared with men, women with heart failure are often older and more likely to have heart failure with preserved ejection fraction with a hypertensive rather than ischemic etiology. Important trends in the incidence and outcomes of heart failure have been recently published. ${ }^{5,6}$ These studies suggest that the incidence of heart failure has declined in many cohorts; however, the prognosis of this disease remains poor. There are known sex-based differences in the risk factors, presentation and management of heart disease. ${ }^{7-9}$ At a time when heart failure management has greatly improved, detailed sex-stratified outcomes are scarce in hospital-admitted cohorts and unavailable in ambulatory patients. Given the sex differences in comorbidities and outcomes in heart failure, it is not known whether and how these current changes are modified by sex. An in-depth understanding of these sex differences may help to increase awareness and lead to clinical trials to identify optimal monitoring and treatment strategies in women and men. We examined the sex differences in heart failure incidence, mortality, hospital admissions and comorbidities in a contemporary populationbased cohort from fiscal years 2009 to 2013. 


\section{Methods}

\section{Study design and population}

We conducted a population-based, retrospective cohort study in Ontario, Canada, using linked administrative databases. Included were patients 40 years of age and older who were newly diagnosed with heart failure in an ambulatory setting between Apr. 1, 2009, and Mar. 31, 2014. We excluded non-Ontario residents, those who were 105 years of age and older at the time of diagnosis, and those diagnosed with heart failure in an inpatient setting. During the study period, Ontario was Canada's most populous province with a publicly funded, universal health care system that reimbursed all medically necessary health care and hospital services.

\section{Data sources}

Databases were linked deterministically using unique encoded identifiers. Incident cases of heart failure were identified using the Institute for Clinical Evaluative Sciences (ICES) Ontario Congestive Heart Failure database, where ambulatory cases of heart failure were derived from Ontario Health Insurance Plan (OHIP) billings based on 1 outpatient claim for heart failure that was followed by at least 1 additional outpatient claim within 1 year. Heart failure diagnoses made during an emergency department visit that did not result in hospital admission were considered ambulatory. This algorithm was validated in primary care patient records and shown to have $85 \%$ sensitivity and $97 \%$ specificity in identifying heart failure events. ${ }^{10}$ Analyses were conducted by linking the ICES Ontario Congestive Heart Failure database with the Registered Persons Database for demographics and vital statistics, the Canadian Institute for Health Information (CIHI) Discharge Abstract Database for hospital admissions and comorbidities, the CIHI Same Day Surgery Database for comorbidities and the OHIP database for physician fee-for-service claims. Although they lack physiologic and laboratory measures, these databases have been validated for the measurement of many outcomes, exposures and comorbidities. ${ }^{11-14}$

\section{Outcomes}

The primary outcome was all-cause mortality within 1 year of the diagnosis of heart failure. Mortality was ascertained by using the Registered Persons Database. The secondary outcome was hospital admission for heart failure within 1 year of the diagnosis, which was ascertained by using the Discharge Abstract Database.

\section{Covariates}

Demographic variables (i.e., age and sex) were obtained from the Registered Persons Database. We estimated socioeconomic status based on patients' neighbourhood median income in the Canadian census, and determined their residence (rural v. urban) using definitions from Statistics Canada. ${ }^{15}$ We identified hypertension, ${ }^{11}$ asthma, ${ }^{16}$ chronic obstructive pulmonary disease $(C O P D)^{17}$ and diabetes mellitus ${ }^{13}$ using validated algorithms. Other comorbidities were identified using the Discharge Abstract Database, Same Day Surgery Database and OHIP databases based on International Statistical Classification of Diseases and
Related Health Problems, 10th revision, codes within 5 years before the heart failure diagnosis, using previously described methods. ${ }^{18,19}$ Frailty status was identified using the Johns Hopkins Adjusted Clinical Groups frailty-defining diagnoses indicator, which is an instrument designed and validated for research of frailty-related outcomes and resource utilization using administrative data. ${ }^{20-24}$

\section{Statistical analysis}

All analyses were performed in the overall cohort and then stratified by sex. Continuous variables were analyzed using the Student $t$ test and expressed as means and standard deviations. Categorical variables were analyzed using the $\chi^{2}$ test and expressed as numbers and proportions. Standardized differences were used to compare the characteristics of men and women with incident heart failure. ${ }^{25}$

Survival time was defined from the date of heart failure diagnosis until death or last follow-up. Patients were censored when they lost possession of valid Ontario health insurance for 2 consecutive eligibility quarters (i.e., they left Ontario). Probability of death was calculated using the Kaplan-Meier method, with the significance of the difference between sexes assessed using the log-rank test. The relative hazard of death was assessed using Cox proportional hazard models with multivariable adjustment for all variables listed in Appendix 1, Supplemental Table 2 (available at www.cmaj.ca/lookup/suppl/doi:10.1503/cmaj.180177/-/ DC1), and without the use of selection algorithms. We estimated the cumulative incidence of hospital admissions for heart failure using the cumulative incidence function, which treated death as a competing risk. Rates were directly standardized by age using the 1991 Canadian population as the reference. To justify sexspecific analyses, we tested for the presence of any interaction between sex and each of the mortality risk factors using multiplicative interaction terms. The measure of association was hazard ratios (HRs) with 95\% confidence intervals ( $\mathrm{Cls}$ ). Analyses were performed using SAS version 9.3, with statistical significance defined by a 2 -sided $p$ value less than 0.05 .

\section{Ethics approval}

The research ethics board of the Sunnybrook Health Sciences Centre approved this study and waived the need for informed consent.

\section{Results}

A total of 90707 diagnoses of heart failure were made in an ambulatory setting during the 5 -year study period (47\% women). Compared with male patients, female patients with heart failure were more likely to be older and more frail, to have lower socioeconomic status, and to have comorbid conditions such as hypertension, pulmonary circulatory disease, COPD, hypothyroidism, metastatic malignancy, dementia and depression. Conversely, women were less likely than men to have atrial fibrillation, myocardial infarction (MI), valvular disease, peripheral arterial disease, diabetes, renal disease, liver disease and alcohol abuse (Table 1). 
Heart failure incidence, mortality and hospital admission

Heart failure incidence reached a nadir for both men and women in fiscal years 2011 and 2012, but began to rebound in 2013 (Figure 1; Appendix 1, Supplemental Table 1).

A total of 7156 women (16.8\%) and 7138 men (14.9\%) died within 1 year of follow-up. Age-standardized mortality rates decreased in both sexes, but remained higher in women than in men. Specifically, the female age-standardized mortality rate per 1000 was $89(95 \% \mathrm{Cl} 80-100)$ in 2009 and $85(95 \% \mathrm{Cl} 75-95)$ in
2013 , and the male age-standardized mortality rate was $88(95 \%$ $\mathrm{Cl} 80-97)$ in 2009 and 83 (95\% Cl 75-91) in 2013 (Appendix 1, Supplemental Table 1). Kaplan-Meier survival curves show lower estimated survival in women compared with men (Figure 2A). The overlapping $\mathrm{Cls}$ in the male and female age-standardized mortality rates preclude our ability to conclude on the significance of the relation between sex and mortality over time.

Trends of incident hospital admissions for heart failure reversed in men and women over the course of the study (Appendix 1, Supplemental Table 1). Specifically, the age-standardized

\section{Table 1: Characteristics of 90707 patients with incident heart failure}

\begin{tabular}{|c|c|c|c|c|}
\hline \multirow[b]{2}{*}{ Characteristic } & \multicolumn{2}{|c|}{ No. $(\%)$ of patients* } & \multirow[b]{2}{*}{$p$ value } & \multirow[b]{2}{*}{$\begin{array}{c}\text { Absolute standardized } \\
\text { difference }\end{array}$} \\
\hline & $\begin{array}{c}\text { Women } \\
n=42746\end{array}$ & $\begin{array}{c}\text { Men } \\
n=47961\end{array}$ & & \\
\hline Age at diagnosis, $y r$, mean \pm SD & $75.8 \pm 12.9$ & $71.5 \pm 12.8$ & $<0.001$ & 0.33 \\
\hline Rural residence & $5358(12.5)$ & $6788(14.2)$ & $<0.001$ & 0.05 \\
\hline \multicolumn{5}{|l|}{ Income quintile† } \\
\hline 1 (lowest) & $9613(22.5)$ & $9541(19.9)$ & $<0.001$ & 0.06 \\
\hline 2 & $9243(21.6)$ & $9833(20.5)$ & & 0.03 \\
\hline 3 & 8325 (19.5) & $9444(19.7)$ & & 0.01 \\
\hline 4 & $8017(18.8)$ & $9775(20.4)$ & & 0.04 \\
\hline 5 (highest) & $7388(17.3)$ & $9188(19.2)$ & & 0.05 \\
\hline \multicolumn{5}{|l|}{ Comorbidities } \\
\hline Benign hypertension & $32809(76.8)$ & $34103(71.1)$ & $<0.001$ & 0.13 \\
\hline Complicated hypertension & $5669(13.3)$ & $6516(13.6)$ & 0.2 & 0.01 \\
\hline Atrial fibrillation & $5957(13.9)$ & $6919(14.4)$ & 0.04 & 0.01 \\
\hline Myocardial infarction & $5051(11.8)$ & $9617(20.1)$ & $<0.001$ & 0.23 \\
\hline Valvular disease & $2099(4.9)$ & $2841(5.9)$ & $<0.001$ & 0.04 \\
\hline Peripheral arterial disease & $1272(3.0)$ & $2257(4.7)$ & $<0.001$ & 0.09 \\
\hline Cerebrovascular disease & $2506(5.9)$ & $2713(5.7)$ & 0.2 & 0.01 \\
\hline Pulmonary circulatory disease & $1113(2.6)$ & $882(1.8)$ & $<0.001$ & 0.05 \\
\hline COPD or asthma & $16261(38.0)$ & $17164(35.8)$ & $<0.001$ & 0.05 \\
\hline Alcohol abuse & $337(0.8)$ & $1123(2.3)$ & $<0.001$ & 0.13 \\
\hline Renal disease & $1954(4.6)$ & $2865(6.0)$ & $<0.001$ & 0.06 \\
\hline Diabetes & $14751(34.5)$ & $18721(39.0)$ & $<0.001$ & 0.09 \\
\hline Hypothyroidism & $1300(3.0)$ & $479(1.0)$ & $<0.001$ & 0.15 \\
\hline Liver disease & $579(1.4)$ & $895(1.9)$ & $<0.001$ & 0.04 \\
\hline Dementia & $1791(4.2)$ & $1194(2.5)$ & $<0.001$ & 0.09 \\
\hline Depression & $1213(2.8)$ & $756(1.6)$ & $<0.001$ & 0.09 \\
\hline Psychosis & $223(0.5)$ & $171(0.4)$ & $<0.001$ & 0.02 \\
\hline Primary tumour & $3376(7.9)$ & $4419(9.2)$ & $<0.001$ & 0.05 \\
\hline Metastatic cancer & $1000(2.3)$ & $840(1.8)$ & $<0.001$ & 0.04 \\
\hline Paraplegia & $434(1.0)$ & $475(1.0)$ & 0.7 & 0.003 \\
\hline Venous thromboembolism & $450(1.1)$ & $512(1.1)$ & 0.8 & 0.001 \\
\hline Frailty & $11223(26.3)$ & $8409(17.5)$ & $<0.001$ & 0.21 \\
\hline
\end{tabular}


rate of hospital admission for heart failure per 1000 was higher in men (rate 103, 95\% Cl 93-114) than women (rate $90,95 \% \mathrm{Cl}$ 79-101]) in 2009. In 2013, these rates were higher in women (rate 98, 95\% Cl 86-110) than men (rate 91, 95\% Cl 82-101). Cumulative incidence function curves (Figure $2 \mathrm{~B}$ ) illustrate higher cumulative incidence of hospital admissions for heart failure in women compared with men. The overlapping $\mathrm{Cls}$ in the male and female age-standardized rates of hospital admission for heart failure preclude our ability to conclude on the significance of the relation between sex and hospital admissions over time.

\section{The association of sex and 1-year mortality}

Female sex was associated with an increased risk of death in the unadjusted analysis (HR 1.14, 95\% Cl 1.10-1.18). However, it was mildly protective after adjustment for other risk factors listed in Appendix 1, Supplemental Table 2 (adjusted HR 0.97, 95\% Cl 0.93-1.00). Other multivariable predictors of mortality were advanced age, low socioeconomic status, peripheral arterial disease, cerebrovascular disease, COPD or asthma, alcohol abuse, liver disease, renal disease, diabetes, dementia, depression, psychosis, primary and metastatic malignancies, paraplegia and venous thromboembolism (Appendix 1, Supplemental Table 2). Conversely, hypertension and valvular disease were correlated with lower risks of mortality.

Table 2 shows the sex-specific HRs for 1-year mortality in this ambulatory cohort. Of note, low socioeconomic status was associated with a higher risk of mortality, and MI was associated with a lower risk of mortality in men but not in women. In addition, men with COPD or asthma, dementia and cancer had a higher risk of death than women, and women with peripheral arterial disease had a higher risk of death than men.

\section{Interpretation}

We found that, despite modern advances in heart failure therapy, heart failure mortality remains high, especially in women. We found additional sex discrepancies regarding hospital admissions for heart failure and associated comorbidities.

Substantial progress in heart failure management has been made over the past decade. ${ }^{1}$ In addition, risk factors for heart failure have changed owing to advances in the treatment of its leading cause, ischemic heart disease, ${ }^{26}$ and the rising prevalence of obesity and diabetes..$^{27,28}$

In the Framingham Heart Study, researchers evaluated a mixed hospital-admitted and ambulatory cohort from 1950 to 1999 and reported that the incidence of heart failure declined in women but not in men, and survival improved in both sexes. ${ }^{29}$ Another community-based report from 1979 to 2000 found stable heart failure incidence but a greater improvement in survival in men than in women. ${ }^{30}$ Yeung and colleagues observed continued decline in both heart failure incidence and 1-year mortality in ambulatory patients from 1997 to $2007 . .^{5}$ More recently, Christiansen and colleagues reported similar decreases in heart failure incidence and mortality from 1999 to $2012 .{ }^{6}$ Our findings suggest that the incidence of heart failure has declined from 2009 to 2013, but continues to be greater in men than in women. The incidence of heart failure observed in our study is lower than that described in previous studies ${ }^{6,29,30}$ and may reflect a continuing temporal decline of the incidence of heart failure or increasing acuity of heart failure presentations in the modern era (i.e., an increase in the proportion of first heart failure diagnosis in hospital). The latter may also be related to the younger age of our cohort at the time of diagnosis. Specifically, younger patients

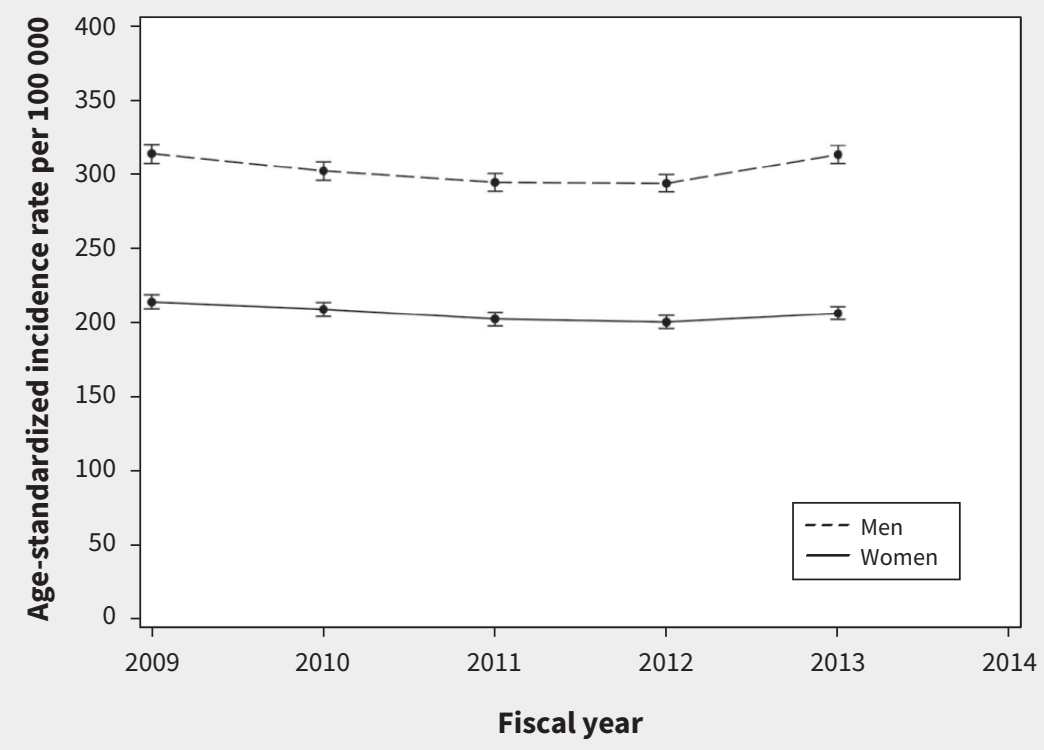

Figure 1: Age-standardized trends in the incidence of heart failure in ambulatory patients. 
may more likely present in an ambulatory care setting, whereas older, sicker patients may more likely present in an acute care setting. Despite improvements in medical therapy, mortality from heart failure remains high. This is particularly relevant given that ambulatory patients are often perceived to be at lower risk compared with inpatients. The age-standardized mortality rates were higher for women than men. However, after multivariable adjustment, female sex was associated with a slightly lower risk of mortality. This observation emphasizes the importance of adjustment for confounding factors in research on heart failure, as well as the evaluation of comorbidities in the management of patients with heart failure.

We observed a reversal of the ratio of male:female hospitaladmission rates over time such that these rates decreased for men but increased for women. This is consistent with the literature $^{31}$ and may reflect death being a competing risk for hospital admission in men. Alternatively, it possibly also reflects the underlying heart failure type, since men are more likely to have

A

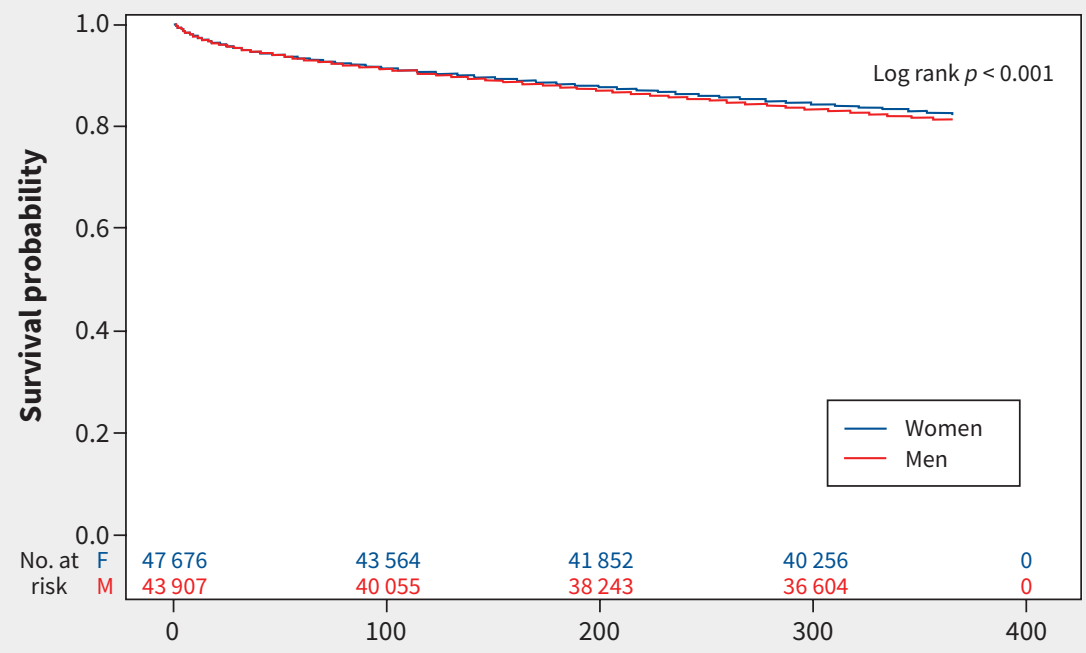

Time, $d$

B

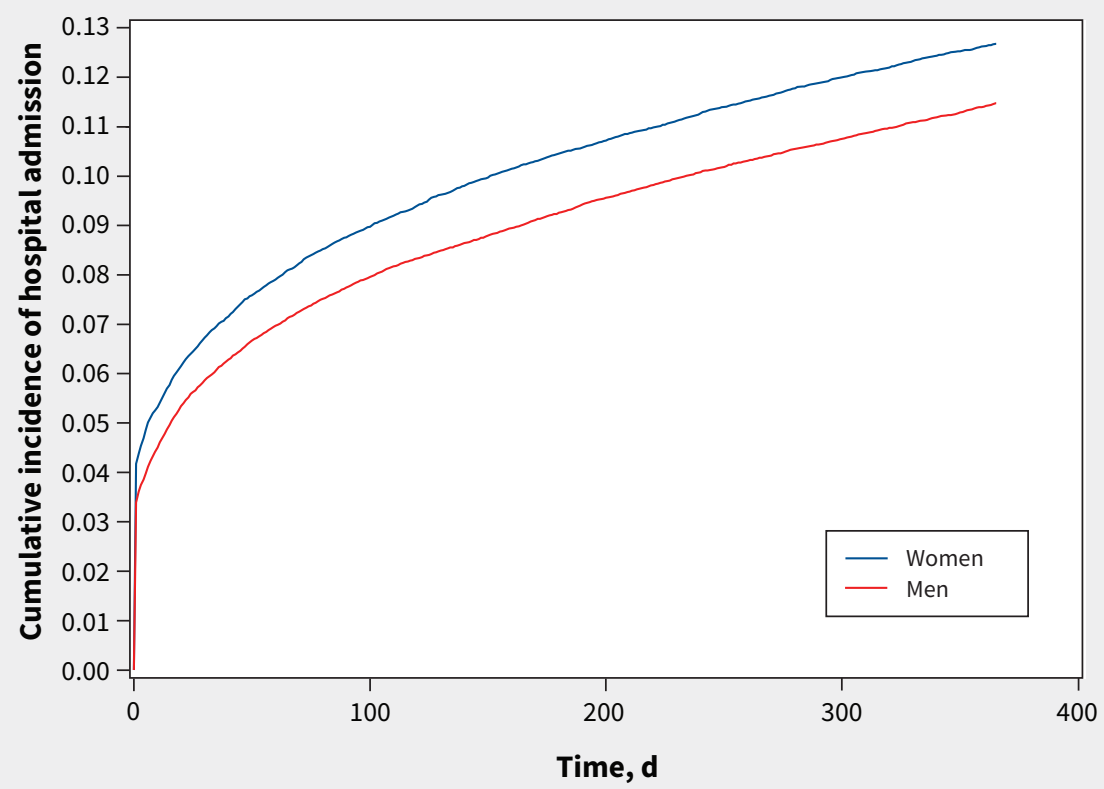

Figure 2: A) Sex differences in 1-year survival after heart failure diagnosis. B) Cumulative incidence of hospital admission for heart failure in women and men within 1 year of diagnosis. 
$\mathrm{HR}(95 \% \mathrm{Cl})$

\begin{tabular}{lc} 
Variable & Women \\
\hline Income quintile & \\
\hline 1 (lowest) & $1.02(0.94-1.09)$ \\
\hline 2 & $0.97(0.90-1.05)$ \\
3 & $0.99(0.92-1.07)$ \\
4 & $1.01(0.93-1.09)$ \\
5 (highest) & Ref. \\
\hline Myocardial infarction & $1.05(0.98-1.12)$ \\
\hline Peripheral arterial disease & $1.48(1.32-1.66)$ \\
COPD or asthma & $1.12(1.07-1.17)$ \\
Dementia & $1.87(1.72-2.02$ \\
\hline Primary tumour & $1.44(1.34-1.56)$ \\
Metastatic malignancy & $3.05(2.75-3.38)$
\end{tabular}

Multiplicative interaction $p$ value*

Note: $\mathrm{Cl}=$ confidence interval, $\mathrm{COPD}=$ chronic obstructive pulmonary disease, $\mathrm{HR}=$ hazard ratio, Ref. $=$ reference.

*Multiplicative interaction terms were formed by multiplying sex by each of the covariates in the multivariable Cox proportional hazard model for 1-year mortality. Only significant interaction terms were reported in this table.

tBased on the patients' neighbourhood median income.

heart failure with reduced ejection fraction (for which there are several treatments known to improve outcomes and decrease hospital admission), whereas women are more likely to have heart failure with preserved ejection fraction ${ }^{32}$ (for which therapies are aimed only at alleviating symptoms, and no therapies have been shown to alter the natural history or outcomes).

A recent report of inpatients with heart failure suggested an inverse relation between left ventricle ejection fraction and adverse outcomes in men but not in women, as well as sex differences in the prognostic impact of comorbidities such as anemia. ${ }^{33}$ These studies suggest that an in-depth understanding of sex differences in comorbidities could inform management decisions. Women with heart failure are older and more likely than men to have hypertension, renal failure, obesity and depression, but overall better survival. Men with heart failure are more often smokers and more likely to have MI, COPD and heart failure with reduced ejection fraction. ${ }^{34}$ Our findings are consistent across many of these comorbidities; however, we found lung disease to be more common in women. Whether this is due to sex differences in smoking rates, and uptake and compliance with smoking cessation programs is not known. In addition, we found frailty, dementia and metastatic malignancy to be more prevalent in women, which likely reflect older ages at heart failure presentation in women. Interestingly, we also found a correlation of hypertension with lower mortality risk in both sexes, and a correlation of MI with lower mortality risk only in men. The protective effect of hypertension may be related to the increased risk associated with low blood pressure in patients with heart failure. ${ }^{35,36}$ Furthermore, women with ischemic heart disease may experience higher rates of missed or delayed management owing to atypical presentation and lower rates of coronary catheterization. The latter may contribute in part to an underestimation of ischemic heart disease severity and poorer outcomes. ${ }^{37,38}$

\section{Limitations}

Our study has several limitations. First, cases of heart failure were identified in the ambulatory care setting based on the requirement of 2 claims for heart failure within 1 year. Although this method may underestimate heart failure, it has been validated and shown to improve the specificity of our case selection. ${ }^{5,10}$ Second, information on ejection fraction was not available in the databases used, which precluded analyses in subtypes of heart failure. Third, owing to the short period of observation, we are unable to comment on important changes in patient characteristics or outcomes during the study period. Fourth, we examined all-cause and not cause-specific mortality. However, similar studies have also reported only on all-cause mortality. Finally, our data sources lacked some relevant details, such as body mass index and smoking. The inability to adjust for the differences in these characteristics could have led to unmeasured confounding.

\section{Conclusion}

We found that mortality from heart failure remains high, especially in women; that hospital admissions for heart failure decreased in men but increased in women; and that women and men had different associated comorbidities. Further studies should focus on sex differences in health-seeking behaviour, medical therapy and response to therapy to improve outcomes in women.

\section{References}

1. Ezekowitz JA, O'Mera E, McDonald MA, et al. 2017 Comprehensive update of the Canadian Cardiovascular Society Guidelines for the Management of Heart Failure. Can J Cardiol 2017;33:1342-433.

2. McMurray JJ, Adamopoulos S, Anker SD, et al. ESC Guidelines for the diagnosis and treatment of acute and chronic heart failure 2012: the Task Force for the Diagnosis and Treatment of Acute and Chronic Heart Failure 2012 of the European Society of Cardiology. Developed in collaboration with the Heart Failure Association (HFA) of the ESC. Eur Heart J 2012;33:1787-847. 
3. Huffman MD, Berry JD, Ning H, et al. Lifetime risk for heart failure among white and black Americans: cardiovascular lifetime risk pooling project. J Am Coll Cardiol 2013;61:1510-7.

4. Roger VL, Go AS, Lloyd-Jones DM, et al. Heart disease and stroke statistics 2011 update: a report from the American Heart Association. Circulation 2011;123:e18-209.

5. Yeung DF, Boom NK, Guo H, et al. Trends in the incidence and outcomes of heart failure in Ontario, Canada: 1997 to 2007. CMAJ 2012;184:E765-73.

6. Christiansen MN, Kober L, Weeke P, et al. Age-specific trends in incidence, mortality, and comorbidities of heart failure in Denmark, 1995 to 2012. Circulation 2017;135:1214-23.

7. Scantlebury DC, Borlaug BA. Why are women more likely than men to develop heart failure with preserved ejection fraction? Curr Opin Cardiol 2011;26:562-8.

8. Dunlay SM, Roger VL. Gender differences in the pathophysiology, clinical presentation, and outcomes of ischemic heart failure. Curr Heart Fail Rep 2012;9:267-76.

9. Kenchaiah S, Vasan RS. Heart failure in women - insights from the Framingham Heart Study. Cardiovasc Drugs Ther 2015;29:377-90.

10. Schultz SE, Rothwell DM, Chen Z, et al. Identifying cases of congestive heart failure from administrative data: a validation study using primary care patient records. Chronic Dis Inj Can 2013;33:160-6.

11. Tu K, Campbell NRC, Chen ZL, et al. Accuracy of administrative databases in identifying patients with hypertension. Open Med 2007;1:e18-26.

12. Juurlink D, Preya C, Croxford R, et al. Canadian Institute for Health Information Discharge Abstract Database: a validation study. ICES investigative report. Toronto: Institute for Clinical Evaluative Sciences; 2006.

13. Hux JE, Ivis F, Flintoft V, et al. Diabetes in Ontario: determination of prevalence and incidence using a validated administrative data algorithm. Diabetes Care 2002;25:512-6.

14. Austin PC, Daly PA, Tu JV. A multicenter study of the coding accuracy of hospital discharge administrative data for patients admitted to cardiac care units in Ontario. Am Heart J 2002;144:290-6.

15. du Plessis V, Beshiri R, Bollman RD, et al. Definitions of "rural." Agriculture and rural working paper series, no. 61. Ottawa: Statistics Canada; 2002

16. Gershon AS, Wang C, Guan J, et al. Identifying patients with physician-diagnosed asthma in health administrative databases. Can Respir J 2009;16:183-8.

17. Gershon AS, Wang C, Guan J, et al. Identifying individuals with physcian diagnosed COPD in health administrative databases. COPD 2009;6:388-94.

18. Quan H, Sundararajan V, Halfon P, et al. Coding algorithms for defining comorbidities in ICD-9-CM and ICD-10 administrative data. Med Care 2005;43:130-9.

19. Sun LY, Gershon AS, Ko DT, et al. Trends in pulmonary function testing before noncardiothoracic surgery. JAMA Intern Med 2015;175:1410-2.

20. Lieberman RAC, Weiner J. Development and evaluation of the Johns Hopkins University risk adjustment models for Medicare+Choice plan payment. Baltimore: Johns Hopkins University; 2003.

21. Sternberg SA, Bentur N, Abrams C, et al. Identifying frail older people using predictive modeling. Am J Manag Care 2012;18:e392-7.
22. The Johns Hopkins adjusted clinical groups technical reference guide. Version 9.0. Baltimore: Johns Hopkins University; 2009.

23. Bronskill SE, Carter MW, Costa AP, et al. Aging in Ontario: an ICES chartbook of health services use by older adults - a technical report. Toronto: Institute for Clinical Evaluative Sciences; 2010.

24. Bronskill SCX, Gruneir A, Ho M. Health system use by frail Ontario seniors: an in-depth examination of four vulnerable cohorts. Toronto: Institute for Clinical Evaluative Sciences; 2011.

25. Mamdani M, Sykora K, Li P, et al. Reader's guide to critical appraisal of cohort studies: 2. Assessing potential for confounding. BMJ 2005;330:960-2.

26. Finegold JA, Asaria P, Francis DP. Mortality from ischaemic heart disease by country, region, and age: statistics from World Health Organisation and United Nations. Int J Cardiol 2013;168:934-45.

27. Cheng S, Claggett B, Correia AW, et al. Temporal trends in the population attributable risk for cardiovascular disease: the Atherosclerosis Risk in Communities study. Circulation 2014;130:820-8.

28. NCD Risk Factor Collaboration (NCD-RisC). Trends in adult body-mass index in 200 countries from 1975 to 2014: a pooled analysis of 1698 population-based measurement studies with 19.2 million participants. Lancet 2016;387:1377-96.

29. Levy D, Kenchaiah S, Larson MG, et al. Long-term trends in the incidence of and survival with heart failure. N Engl J Med 2002;347:1397-402.

30. Roger VL, Weston SA, Redfield MM, et al. Trends in heart failure incidence and survival in a community-based population. JAMA 2004;292:344-50.

31. Ziaeian B, Kominski GF, Ong MK, et al. National differences in trends for heart failure hospitalizations by sex and race/ethnicity. Circ Cardiovasc Qual Outcomes 2017;10: pii:e003552.

32. Bhatia RS, Tu JV, Lee DS, et al. Outcome of heart failure with preserved ejection fraction in a population-based study. N Engl J Med 2006;355:260-9.

33. Kajimoto K, Minami Y, Sato N, et al.; Acute decompensated heart failure syndromes (ATTEND) registry. Gender differences in left ventricular ejection fraction and outcomes among patients hospitalized for acute decompensated heart failure. Am J Cardiol 2017;119:1623-30.

34. Hopper I, Kotecha D, Chin KL, et al. Comorbidities in heart failure: Are there gender differences? Curr Heart Fail Rep 2016;13:1-12.

35. Raphael CE, Whinnett ZI, Davies JE, et al. Quantifying the paradoxical effect of higher systolic blood pressure on mortality in chronic heart failure. Heart 2009;95:56-62.

36. Schmid FA, Schlager O, Keller P, et al. Prognostic value of long-term blood pressure changes in patients with chronic heart failure. Eur $J$ Heart Fail 2017; 19:837-42

37. Jarvie JL, Foody JM. Recognizing and improving health care disparities in the prevention of cardiovascular disease in women. Curr Cardiol Rep 2010; 12:488-96.

38. McSweeney J, Pettey C, Lefler LL, et al. Disparities in heart failure and othe cardiovascular diseases among women. Womens Health (Lond Engl) 2012; 8:473-85.

\section{Competing interests: None declared.}

This article has been peer reviewed.

Affiliations: Division of Cardiac Anesthesiology, Department of Anesthesiology and Pain Medicine (Sun), University of Ottawa Heart Institute, Ottawa, Ont.; Institute for Clinical Evaluative Sciences (Sun, Tu, Bader Eddeen); Division of Cardiology (Tu), Schulich Heart Centre, Sunnybrook Health Sciences Centre, University of Toronto, Toronto, Ont:; Division of Cardiology, Department of Medicine (Coutinho, Mielniczuk), University of Ottawa Heart Institute; Division of Cardiac Prevention and Rehabilitation (Coutinho, McDonnell, Tulloch), University of Ottawa Heart Institute; Division of Cardiology, Department of Medicine (Turek), The Ottawa Hospital; Division of Cardiac Surgery, Department of Surgery (Rubens), University of Ottawa Heart Institute, Ottawa, Ont.

Contributors: Louise Sun and Lisa Mielniczuk contributed to the conception and design of the work, and obtained funding for the study. Jack Tu contributed to the design of the work. Louise Sun and Anan Bader Eddeen contributed to the acquisition and analysis of data. All of the authors contributed to the interpretation of data. Louise Sun and Lisa Mielniczuk contributed to drafting the manuscript, which all of the authors revised. All of the authors (with the exception of Jack Tu) gave final approval of the version to be published and agreed to be accountable for all aspects of the work, including accuracy and integrity of the data presented.

*Dr. Jack Tu passed away on May 30, 2018, during preparation of this manuscript for publication.

Funding: This work was supported by a team grant from the University of Ottawa Heart Institute (grant \#4554). Jack Tu was supported by a Canada Research Chair in Health Services Research and an Eaton Scholar Researcher of the Year Award from the Department of Medi- cine, University of Toronto. Thais Coutinho holds the University of Ottawa Heart Institute's Chair in Women's Heart Health and is a Clinician Scientist supported by a Heart and Stroke Foundation of Ontario Clinician Scientist Phase I Award. Lisa Mielniczuk holds a University of Ottawa Chair in Heart Failure and is supported as a Clinician Scientist from the Heart and Stroke Foundation of Ontario.

Disclaimer: This study was supported by the Institute for Clinical Evaluative Sciences (ICES), which is funded by an annual grant from the Ontario Ministry of Health and Long-Term Care (MOHLTC). The opinions, results and conclusions reported in this paper are those of the authors and are independent from the funding sources. No endorsement by ICES or the Ontario MOHLTC is intended or should be inferred.

Accepted: Apr. 29, 2018.

Correspondence to: Louise Sun, Isun@ ottawaheart.ca 